\title{
Simetria nasal após a realização de rinoplastia associada à queiloplastia em crianças com fissura labial e labiopalatal
}

\author{
Nasal symmetry after rhinoplasty associated with cheiloplasty in cleft lip and \\ palate children
}

Henrique Lopes Arantes ${ }^{1}$

Rafael Ferreira Pereira da

SILVA $^{2}$

José Marcos MélegA ${ }^{3}$

Trabalho realizado no Instituto de Cirurgia Plástica Santa Cruz, São Paulo, SP, Brasil.

Artigo submetido pelo SGP (Sistema de Gestão de Publicações) da RBCP.

Artigo recebido: 7/6/2010 Artigo aceito: 15/3/2011

\begin{abstract}
RESUMO
Introdução: A realização da rinoplastia associada à queiloplastia após os dois meses de idade possibilita reduzir as deformidades nasais e facilita correções secundárias. Objetivo: O objetivo deste trabalho é avaliar a simetria nasal após a realização de rinoplastia associada à queiloplastia em crianças com fissura labial e labiopalatal unilateral. Método: Foram avaliados 41 pacientes após os seis meses de cirurgia. Os pacientes foram submetidos à queiloplastia pela técnica de Millard associada à rinoplastia pela técnica de McComb. Utilizando as fotografias do pós-operatório, avaliou-se a simetria nasal entre o lado normal e fissurado. Resultados: A simetria nasal foi considerada excelente em 53,66\% dos casos, bom em $46,34 \%$ e nenhum paciente foi ruim. Conclusão: A avaliação da rinoplastia propiciou bons e excelentes resultados.
\end{abstract}

Descritores: Rinoplastia. Nariz/anormalidades/cirurgia. Fenda Labial/cirurgia. Procedimentos Cirúrgicos Reconstrutivos/métodos.

\begin{abstract}
Introduction: The aim of realize rhinoplasty associated with the cheiloplasty after two months of age is to reduce nasal deformities and facilitate secondary surgeries. Objective: The purpose of this article is to evaluate the nasal symmetry after rhinoplasty associated with cheiloplasty in unilateral cleft lip and palate children. Methods: A total of 41 patients were evaluated after six month of surgery. All had been treated by the technique of Millard for the cheiloplasty, and by the principles of McComb for the rhinoplasty. Through the postoperative photographies of the children were evaluated the nasal symmetry between the normal and cleft side. Results: The evaluation of nasal symmetry was considered excellent in $53.66 \%$, good in $46.34 \%$ and none was poor. Conclusion: The evaluation of rhinoplasty was considered excellent and good.
\end{abstract}

Keywords: Rhinoplasty. Nose/abnormalities/surgery. Cleft Lip/surgery. Fissura Palatina/ surgery. Reconstructive Surgical Procedures/methods.

\footnotetext{
1. Membro Titular da Sociedade Brasileira de Cirurgia Plástica, Médico assistente do Instituto de Cirurgia Plástica Santa Cruz, São Paulo, SP, Brasil.

2. Médico Residente do Instituto de Cirurgia Plástica Santa Cruz, São Paulo, SP, Brasil.

3. Membro Titular da Sociedade Brasileira de Cirurgia Plástica, Regente do Instituto de Cirurgia Plástica Santa Cruz, São Paulo, SP, Brasil.
} 


\section{INTRODUÇÃO}

O tratamento do nariz de pacientes com fissura labial e labiopalatal sempre foi um desafio para os cirurgiões plásticos. O receio de interferir com o crescimento das estruturas nasais fez com que a correção fosse realizada somente após o desenvolvimento completo deste. Consequentemente, essas crianças cresciam com o estigma da deformidade nasal e o tratamento tardio era muito difícil.

Stenström e Oberg ${ }^{1}$ estudaram a patogênese da deformidade nasal em pacientes com fissura labial. Seus estudos demonstraram que a perda da continuidade do esfíncter muscular oral associada a uma tração lateral pelos músculos faciais explicava a posição anômala da columela, septo nasal, base da asa nasal e alteração do contorno da cartilagem alar maior no lado fissurado.

As primeiras técnicas cirúrgicas descritas para correção de fissura labial se focavam apenas no defeito do lábio, não se importando com as alterações nasais associadas. Com o desenvolvimento dos tratamentos cirúrgicos, ficou evidente a necessidade de corrigir as deformidades nasais, uma vez que, por melhor que fosse o resultado do reparo labial, o desequilíbrio causado na face pelas alterações nasais era um problema considerável ${ }^{2}$.

Blair $^{3}$ e Brown \& McDowell ${ }^{4}$ foram os pioneiros no tratamento do nariz de fissurados e plantaram as primeiras sementes para a realização de rinoplastia associada à queiloplastia.

Brown e McDowell ${ }^{4}$ e Steffensen ${ }^{5}$ preconizaram a realização de incisões internas e externas na abertura narinária e rotação medial da cartilagem alar maior, além de remoção do suposto excesso da cartilagem. Porém os resultados não eram satisfatórios, uma vez que não havia excesso de cartilagem e sim um malposicionamento destas, além disso, não havia boa simetria nasal e as cicatrizes eram conspícuas.

Em geral, os primeiros resultados das rinoplastias associadas às queiloplastias foram insatisfatórios, principalmente porque se realizavam incisões extensas ao longo do contorno da narina, associadas a incisões intercartilaginosas, ou incisões externas no nariz, ao redor das cartilagens alares maiores. Consequentemente, muitos pacientes apresentavam estenoses e deformidades narinárias devido às retrações cicatriciais, contribuindo para que a realização da rinoplastia primária fosse muito questionada.

Berkeley $^{6}$, um grande pesquisador das características nasais dos pacientes fissurados, verificou que a rinoplastia tardia raramente apresentava um resultado satisfatório do ponto de vista funcional e estético.

Anastassov et al. $^{7}$ avaliaram pela rinomanometria e análises geométricas da face 54 pacientes submetidos à correção do nariz tardiamente. Foram verificados distúrbios olfatórios e da permeabilidade nasal na maioria dos pacientes, além de desenvolvimento inadequado do terço médio da face e do nariz. Os autores concluíram que a rinoplastia deveria ser realizada concomitante à queiloplastia, uma vez que quando realizada tardiamente apresentava resultados estéticos e funcionais ruins.

A realização da rinoplastia concomitantemente à queiloplastia após os dois meses de idade consiste em reconstituir ou reposicionar as estruturas ao seu sítio anatômico normal, minimizando as deformidades secundárias e promovendo melhor desenvolvimento físico e psicossocial para as crianças.

Há diversas técnicas de rinoplastia primária e cada cirurgião deve escolher aquela que melhor se adapte e propicie uma boa correção das deformidades nasais e assimetrias. O resultado final da rinoplastia ocorrerá a longo prazo, principalmente nestes pacientes que poderão ser submetidos a novas cirurgias na face e que ainda irão crescer, portanto, o acompanhamento até a adolescência é muito importante.

O objetivo deste estudo foi avaliar precocemente a simetria nasal após a realização de rinoplastia associada à queiloplastia, em crianças com fissura labial e labiopalatal.

\section{MÉTODO}

Foram incluídos no estudo 41 pacientes operados entre fevereiro de 2004 e novembro de 2007, sendo 27 do sexo masculino e 14 do feminino. Os pacientes eram 11 da raça negra, 20 da branca e dez mestiços.

Todas as crianças eram portadoras de fissuras labiais ou labiopalatais unilaterais não sindrômicas, sendo que 15 apresentavam fissuras labiais e 26 labiopalatais. Todas as malformações foram corrigidas (lábio, nariz e palato).

Os pais ou responsável legal pelos pacientes foram informados minuciosamente sobre o tratamento, previamente à cirurgia, e assinaram um termo de consentimento autorizando a cirurgia, o estudo do caso e as fotos pré e pós-operatórias.

Todos os pacientes foram operados pelo autor principal e acompanhados rotineiramente por, no mínimo, seis meses de pós-operatório.

Os pacientes foram submetidos a queiloplastia associada a rinoplastia a partir dos dois meses de idade, com média de idade na ocasião da cirurgia de 9,2 meses. O paciente mais novo tinha dois meses e o mais velho nove anos.

As queiloplastias foram realizadas pela técnica cirúrgica de Millard tipo 2 (Figura 1), e as rinoplastias seguiram os princípios descritos por McComb e Salyer (Figura 2), associado ao uso pós-operatório de moldes intranasais de silicone para auxiliarem na modelagem e no reposicionamento das estruturas nasais ${ }^{2,8-12}$. Após quatorze dias, retiraram-se os pontos captonados e o molde intranarinário. Não foi realizada septoplastia em nenhum paciente. 


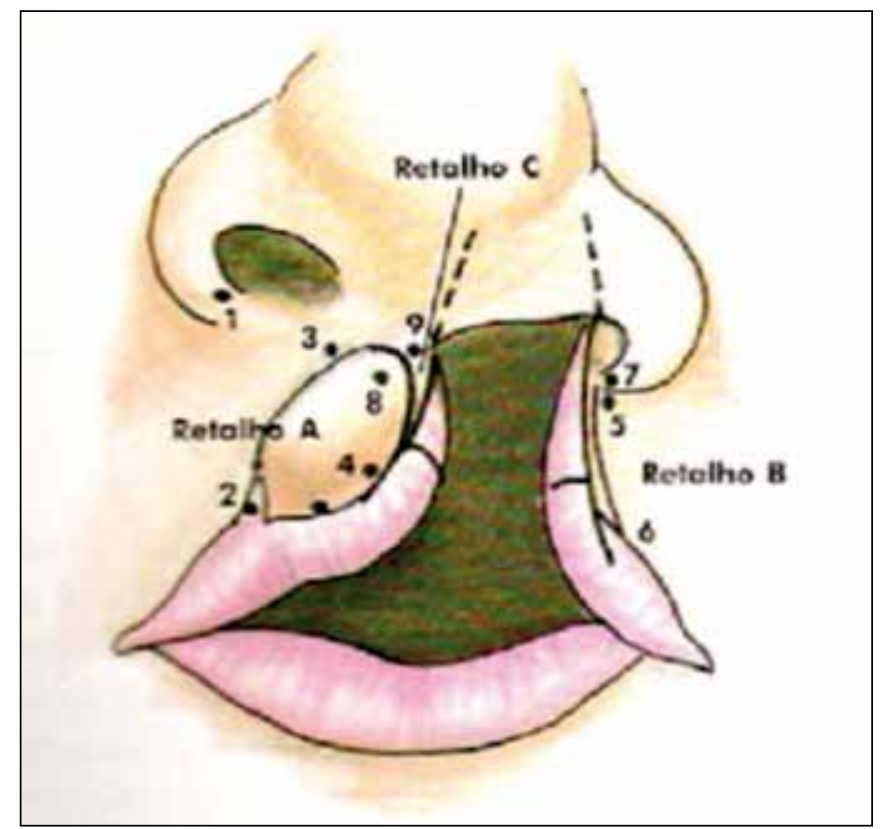

Figura 1-Marcação da queiloplastia pela técnica de Millard.

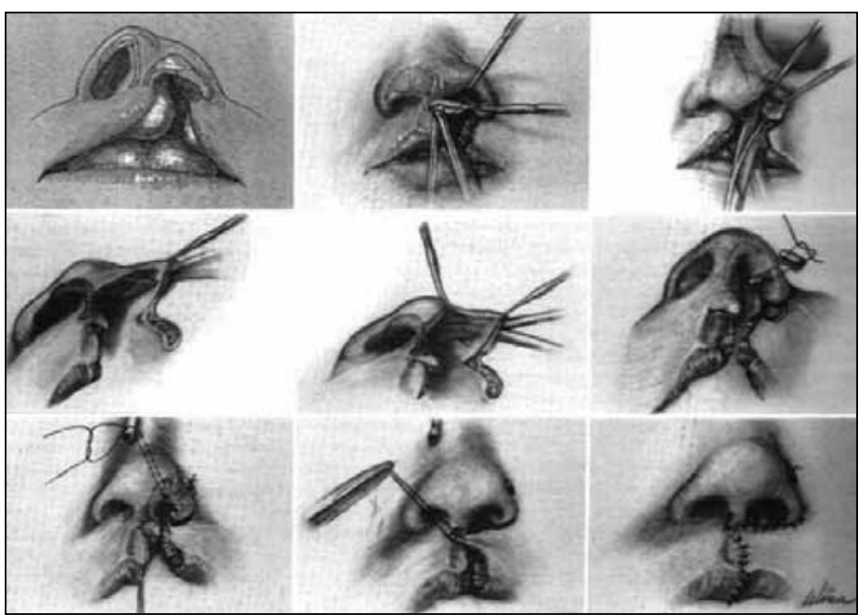

Figura 2 - Rinoplastia primária pela técnica de McComb e Salyer.

Os pacientes foram chamados após seis meses da cirurgia para serem fotografados pelo próprio cirurgião. As fotos foram feitas de frente, perfil direito e esquerdo, semi-perfil direito e esquerdo, e com a cabeça inclinada para trás, para evidenciar as narinas e ápice nasal. Todas as fotos foram realizadas a um metro de distância do paciente. Pelas fotografias, analisou-se a simetria nasal nos planos vertical e horizontal, além do desvio da ponta nasal.

No plano vertical, mensuraram-se os desvios nasais em relação à linha média sagital da face (Figura 3) e também distopias verticais das asas nasais em relação a uma linha interpupilar (Figura 4). Com as fotografias de pós-operatório dos pacientes, traçaram-se as linhas de referência e avaliouse a existência de desvio nasal, calculou-se o ângulo entre o eixo nasal e a linha média sagital e, por fim, mensurou-se a distância entre a base das asas nasal direita e esquerda em relação ao plano da linha interpupilar.

No plano horizontal, calcularam-se as distâncias entre um ponto médio na base da columela e a porção mais lateral das asas nasal direita e esquerda (Figuras 5 e 6$)^{7}$.

A avaliação dos resultados foi feita por uma escala numérica relacionada com as diferenças de medidas, em milímetros $(\mathrm{mm})$, entre o lado normal e o fissurado. A somatória das diferenças de medidas tanto no plano vertical como no horizontal resultava em um número que permitiu a classificação de acordo com uma tabela. Portanto, quando observada uma diferença de um milímetro no plano vertical e um milímetro no horizontal, somava-se 1+1 e obtinha-se a avaliação do resultado ${ }^{7}$.

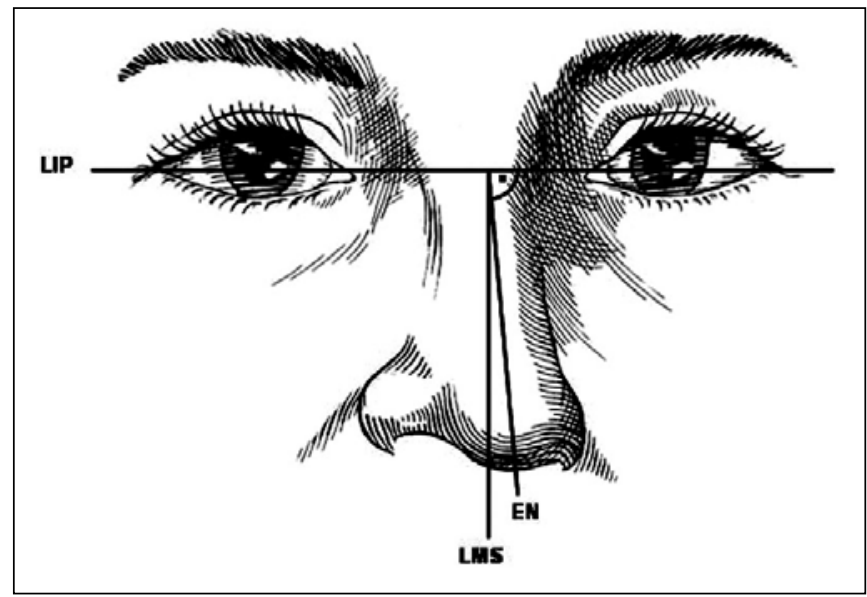

Figura 3 - Desvio do eixo nasal: LIP = linha interpupilar, $L M S=$ linha mediosagital, EN = eixo nasal. $O$ ângulo formado entre LMS e EN é o desvio nasal em relação ao plano vertical.

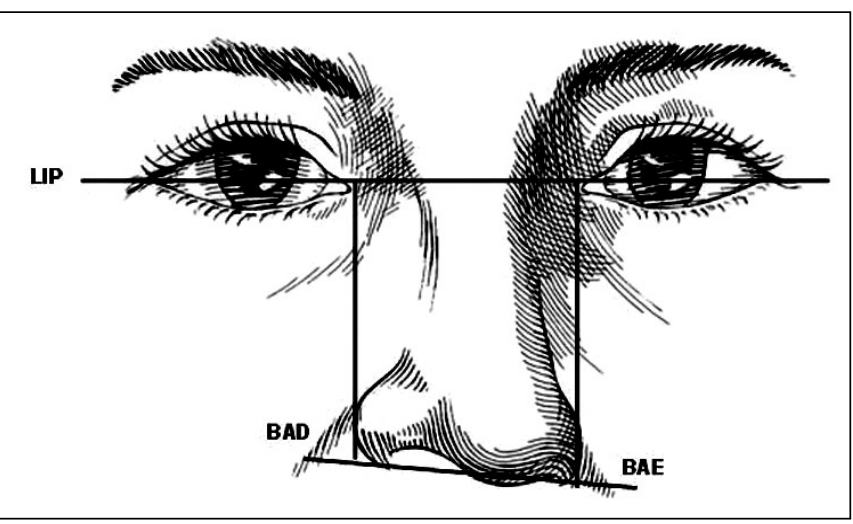

Figura 4 - Posição vertical das bases das asas nasais: LIP = linha interpupilar, $B A D=$ base da asa nasal direita, $B A E=$ base da asa nasal esquerda. 


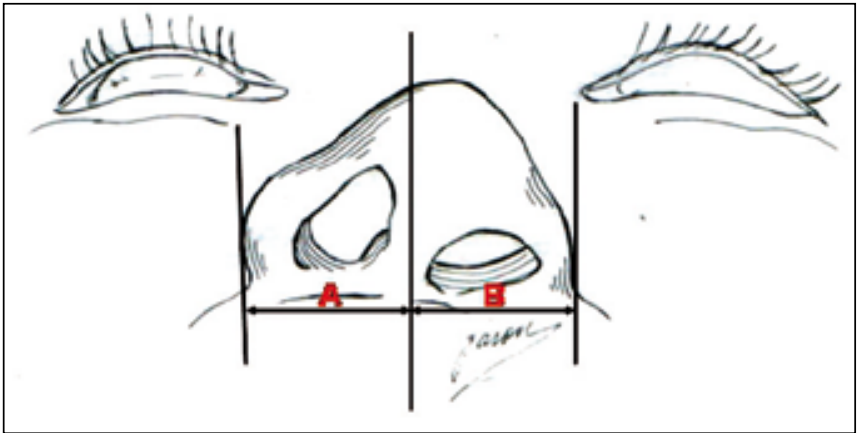

Figura 5 - Avaliação no plano horizontal. A = distância da asa nasal direita à linha média columelar (LMC). $B=$ distância da asa nasal esquerda à linha média columelar.
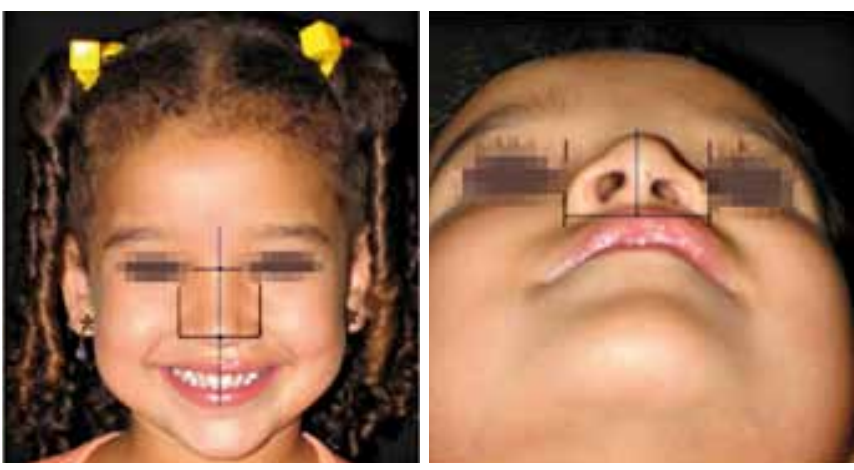

Figura 6 - Avaliação da simetria nasal.

\section{RESULTADOS}

A Tabela 1 apresenta a avaliação dos resultados pela somatória das diferenças de medidas entre o lado fissurado e o normal.

$\mathrm{Na}$ Tabela 2, encontram-se os resultados obtidos com a cirurgia segundo o tipo de fissura.

Os pacientes apresentaram boa simetria e contorno do nariz, melhora da projeção da ponta nasal e, principalmente, da retroposição e alongamento da narina no lado fissurado. Outra característica do nariz de fissurados que melhorou foi o desvio do eixo nasal em relação à linha médio-sagital, sendo

Tabela 1 - Avaliação dos resultados pela somatória das diferenças de medidas entre o lado fissurado e o normal.

\begin{tabular}{c|c}
\hline Somatória & Avaliação \\
\hline 0 até 1 & excelente \\
\hline 2 até 4 & bom \\
\hline$>4$ & ruim \\
\hline
\end{tabular}

\begin{tabular}{|c|c|c|c|c|}
\hline \multirow[b]{2}{*}{$\begin{array}{l}\text { Tipo de } \\
\text { Fissura }\end{array}$} & \multicolumn{4}{|c|}{ Resultados } \\
\hline & $\begin{array}{c}\text { Excelente } \\
(0 \text { até 1) } \\
\text { N (\%) }\end{array}$ & $\begin{array}{c}\text { Bom } \\
(2 \text { até 4) } \\
\text { N (\%) }\end{array}$ & $\begin{array}{c}\text { Ruim } \\
(>4) \\
\text { N (\%) }\end{array}$ & $\begin{array}{l}\text { Total } \\
\text { N (\%) } \\
\end{array}$ \\
\hline Labial & $9(60 \%)$ & $6(40 \%)$ & - & $15(100 \%)$ \\
\hline Labiopalatal & $13(50 \%)$ & $13(50 \%)$ & $\overline{-}$ & $26(100 \%)$ \\
\hline Total & $22(53,6 \%)$ & $19(46,4 \%)$ & - & $41(100 \%)$ \\
\hline
\end{tabular}

que metade da amostra estudada não apresentava qualquer desvio e os demais apresentaram pequenas alterações que variaram entre três e dez graus.

Não foi observada qualquer complicação relacionada à rinoplastia durante o período estudado.

A Figura 7 apresenta alguns casos clínicos, exibindo fotos pré e pós-operatórias.
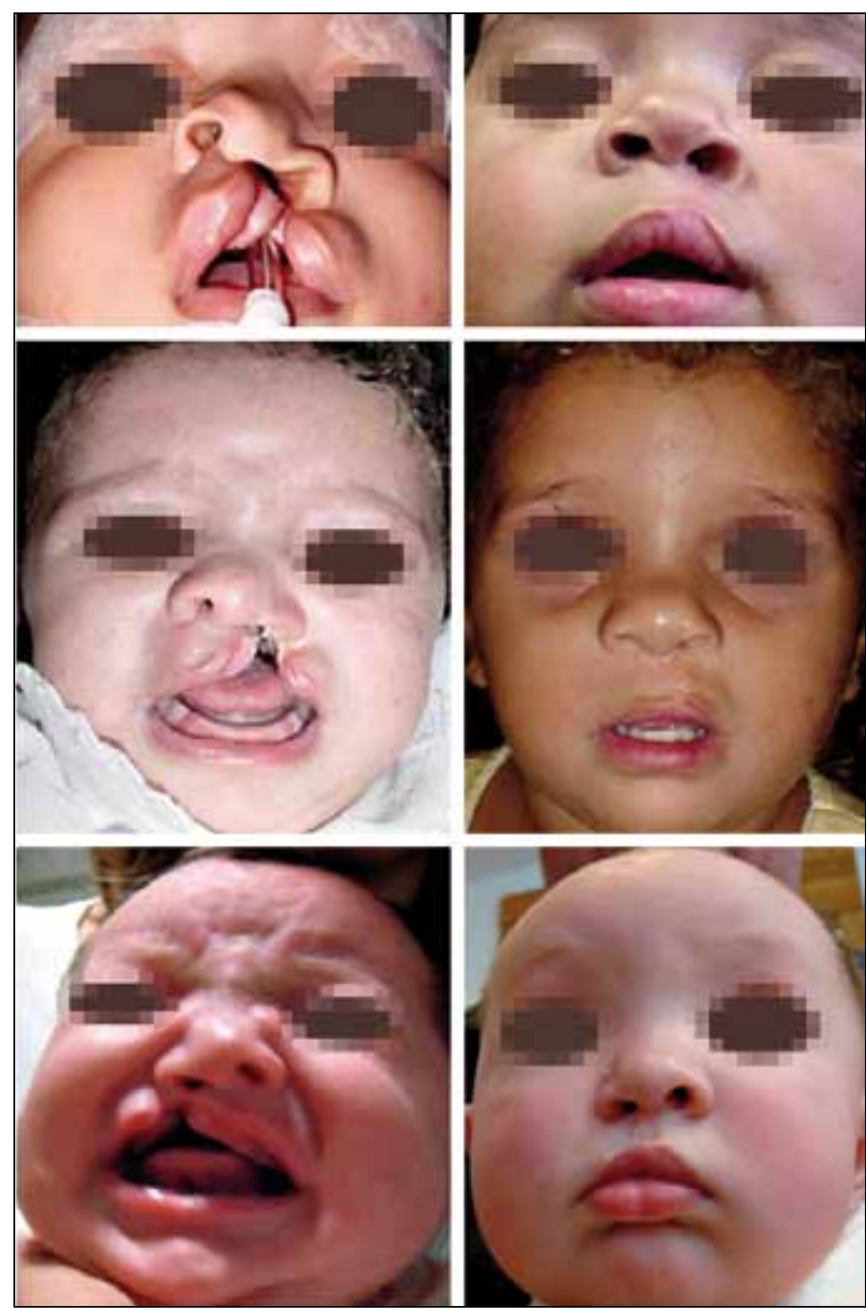

Figura 7-Casos clínicos: pré e pós-operatório. 


\section{DISCUSSÃO}

McComb apresentou sua técnica de rinoplastia primária pela primeira vez no Sixth International Congress of Plastic and Reconstructive Surgery, em 1975, causando muita discussão, pois vários cirurgiões acreditavam que ocorria um prejuízo do crescimento nasal nessas crianças. McComb acompanhou seus pacientes e, após dez anos, em 1985, publicou os resultados satisfatórios dos casos submetidos à rinoplastia associada à queiloplastia. Posteriormente, vários estudos demonstraram que não ocorria qualquer alteração no desenvolvimento da face, tornando essa cirurgia padrão em vários centros de tratamento de pacientes com fissura labiopalatal ${ }^{2,6,8,13-19}$.

A correção precoce do nariz possibilita o reposicionamento das cartilagens alares maiores, melhor projeção do ápice nasal e do contorno das narinas. Todavia, com o crescimento do paciente, pode ser necessário realizar correções secundárias para tornar o nariz mais harmonioso e simétrico.

O maior benefício da rinoplastia primária seria uma possível redução dos estigmas dos pacientes fissurados antes do período escolar, podendo evitar alguns traumas psicológicos durante esta fase vulnerável da criança. Além disso, facilita correções secundárias do nariz após a adolescência ${ }^{20}$.

Huffman and Lierle ${ }^{21}$ demonstraram que o crescimento e a função do nariz ocorrem normalmente quando os tecidos são posicionados de forma correta.

$\mathrm{McComb}^{8,15}$, Salyer ${ }^{16}$, Berkeley et al..$^{6}$ e Boo-Chai ${ }^{19}$ realizaram rinoplastia primária em seus pacientes e os acompanharam até a adolescência, não observando interferência no crescimento do nariz.

A rinoplastia primária pode ser realizada através de incisões externas na columela, intercartilaginosas, marginais ou por meio das incisões nos lábios e narina, associadas ou não a métodos de moldagem pré-operatória ${ }^{20}$. Independente da técnica, deve-se evitar a transecção das cartilagens e o descolamento completo da pele e mucosas, para que não ocorra prejuízo da vascularização e, consequentemente, déficit no desenvolvimento, uma vez que as cartilagens são muito delicadas e frágeis na infância.

Neste grupo de pacientes estudados, verificou-se que a rinoplastia primária não corrige todas as deformidades, observando-se um nariz harmonioso apenas em número reduzido de pacientes, principalmente porque um nariz com medidas simétricas não implica em ser um nariz bonito. No entanto, foi possível corrigir as alterações mais graves, obtendo-se resultados satisfatórios. Sabe-se que seis meses de acompanhamento pós-operatório é pouco tempo para uma avaliação definitiva, mas o objetivo é reduzir as deformidades sem comprometer o crescimento, para que o desenvolvimento seja mais próximo ao do lado normal, minimizando defeitos futuros.

As complicações observadas neste estudo foram relacionadas à queiloplastia e são compatíveis com a literatura ${ }^{15-21}$.

Devido à heterogeneidade da amostra estudada e às variações nas deformidades nasais, seria muito difícil comparar os resultados com um grupo controle não submetido à rinoplastia. Portanto, foi realizado um estudo clínico prospectivo comparando o lado fissurado com o lado normal do próprio paciente. A tabela utilizada permitiu uma análise numérica simplificada dos resultados a partir das fotos de pós-operatório.

Para o futuro, espera-se acompanhar esses pacientes até a adolescência, para avaliarmos o crescimento nasal e a porcentagem de cirurgias para retoques no nariz.

\section{CONCLUSÃo}

A avaliação precoce da simetria nasal em crianças com fissura labial e labiopalatal submetidas à rinoplastia associada à queiloplastia propiciou bons e excelentes resultados.

\section{REFERÊNCIAS}

1. Stenström SJ, Oberg TR. The nasal deformity in unilateral cleft lip. Some notes on its anatomic bases and secondary operative treatment. Plast Reconstr Surg Transplant Bull. 1961;28:295-305.

2. Salyer KE, Genecov ER, Genecov DG. Unilateral cleft lipnose repair: a 33-year experience. J Craniofac Surg. 2003; 14(4):549-58.

3. Blair VP. Nasal deformities associated with congenital cleft of lip. JAMA. 1925;84:185.

4. Brown JB, McDowell F. Secondary repair of cleft lips and their nasal deformities. Ann Surg. 1941;114(1):101-17.

5. Steffensen WH. A method for repair of the unilateral cleft lip. Plast Reconstr Surg. 1949;4(2):144-52.

6. Berkeley WT. Correction of the unilateral cleft lip nasal deformity. In: Grabb WC, Rosenstein SW, Bzoch KR, eds. Cleft lip and palate: surgical, dental and speech aspects. $1^{\mathrm{a}} \mathrm{ed}$. Boston: Little, Brown and Company;1971. p.227-44.

7. Anastassov GE, Joos U, Zöllner B. Evaluation of the results of delayed rhinoplasty in cleft lip and palate patients. Functional and aesthetic implications and factors that affect successful nasal repair. Br J Oral Maxillofac Surg. 1998;36(6):416-24.

8. McComb H. Primary correction of unilateral cleft lip nasal deformity: a 10-year review. Plast Reconstr Surg. 1985;75(6):791-9.

9. McComb H. Treatment of the unilateral cleft lip nose. Plast Reconstr Surg. 1975;55(5):596-601.

10. Millard DR Jr. Earlier correction of the unilateral cleft lip nose. Plast Reconstr Surg. 1982;70(1):64-73.

11. Millard DR Jr., Morovic CG. Primary unilateral cleft nose correction: a 10-year follow-up. Plast Reconstr Surg. 1998;102(5) 1331-8.

12. Millard DR Jr. Cleft craft: the evolution of its surgery. Boston:Little,Brown; 1976.

13. Sundine MJ, Phillips JH. Treatment of the unilateral cleft lip nasal deformity. J Craniofac Surg. 2004;15(1):69-77.

14. Ortiz-Monasterio F, Olmedo A. Corrective rhinoplasty before puberty: a long-term follow-up. Plast Reconstr Surg. $1981 ; 68(3): 381-91$. 
15. McComb H. Primary repair of the bilateral cleft lip nose: a 10-year review. Plast Reconstr Surg. 1986;77(5):701-16.

16. Salyer KE. Primary correction of the unilateral cleft lip nose: a 15 -year experience. Plast Reconstr Surg. 1986;77(4):558-68.

17. Fonseca JLS. Tratamento primário da deformidadenasal do fissurado. In: Mélega JM, ed. Cirurgia plástica fundamentos e arte:cirurgia reparadora de cabeça e pescoço. São Paulo:Medsi;2002. p.166-77.

18. Gomes PRM, Ávila E, Mélega JM. Nariz de fissurado. In: Mélega JM, ed. Cirurgia plástica fundamentos e arte:cirurgia reparadora de cabeça e pescoço. São Paulo:Medsi;2002. p. 197-212.

19. Boo-Chai K. Primary repair of the unilateral cleft lip nose in the Oriental: a 20-year follow-up. Plast Reconstr Surg. 1987;80(2):185-94.

20. Cutting CB, Kamdar MR. Primary bilateral cleft nasal repair. Plast Reconstr Surg. 2008;122(3):918-9.

21. Huffman WC, Lierle DM. Studies on the pathologic anatomy of the unilateral harelip nose. Plast Reconstr Surg. 1949;4(3):225-34.

\section{Correspondência para:}

Rafael Ferreira Pereira da Silva

Av. Doutor Gentil de Moura, 856, apto 13 - Ipiranga - São Paulo, SP, Brasil - CEP 04278-000

E-mail: rafafps04@yahoo.com.br 